\title{
Planetary ionospheres - sources and dynamic drivers
}

\author{
Joseph M. Grebowsky ${ }^{1}$ and Arthur C. Aikin ${ }^{2}$ \\ ${ }^{1}$ NASA Goddard Space Flight Center, \\ Greenbelt, Maryland, USA \\ email: joseph.m.grebowsky@nasa.gov \\ ${ }^{2}$ Institute for Astrophysics and Computational Sciences, \\ Department of Physics, Catholic University of America, \\ Washington, DC, USA \\ email: aaikin1@verizon.net
}

\begin{abstract}
External energy inputs into all planetary upper atmospheres (including more than a half dozen moons with atmospheres) are comprised of combinations of solar EUV, soft x-rays, solar energetic particles, solar wind charged particles, magnetospherically accelerated particles, solar wind electric field, interplanetary dust particles as well as propagating lower atmosphere disturbances. Each input has analogous physical interactions with all planetary ionospheres and upper atmospheres, but the integrated consequences of the multiple energy inputs vary from planet to planet. The Earth forms the framework for most fundamental processes because of extensive measurements of the effects of each of the inputs. However the conditions at Earth are far different from those at the carbon dioxide atmosphere of magnetic field-free, slow-rotating Venus, the carbon dioxide atmosphere of Mars with patchy remnant magnetic fields, while the outer planets have hydrogen atmospheres, are fast rotating with intrinsic magnetic fields, and encompass moons that interact with the magnetospheres and have exotic atmospheres. Although the physical processes are known, our understanding of our solar system's ionospheres diminishes with increasing distance from the Sun.
\end{abstract}

Keywords. earth, meteors, meteoroids, planets and satellites: general,magnetic fields

\section{Introduction}

The uppermost atmospheric layers of all atmospheric-laden planets and moons comprise typically less than one part in a million of the mass of the total atmosphere but these are the planetary boundary regions where energy inputs from interplanetary space and planetary magnetospheres are deposited. The solar wind/magnetosphere energy inputs in all cases produce distinct ionospheric layers and drive motions of the neutral atmosphere that both drag, and are dragged by, the ionospheric ions. Although ionospheric layers are prominent structures often studied by themselves, separate from the atmosphere, their charged particle maximum concentrations are generally much less that those of the neutral atmospheres in the same region. Hence in the main layer of the ionosphere the atmospheric gases and ionization are inseparable parts of the physics. The neutral atmosphere comprises the parent neutrals for the ionized particles responsible for the ionospheric ion composition and the location of the peak ionization. The global topology and motions of the atmosphere and ionosphere are inextricably tied to one another by collisions. All the principle processes controlling the ionization layers throughout the solar system are in general known, lacking still, however, is an understanding of the details of the energy inputs and how different combinations of these inputs produce uniquely different ionospheric variability from one atmosphere to atmosphere. Our knowledge of the 
ionospheric complexities decrease as one moves away from Earth and is perhaps weakest for the atmospheric-laden moons of the outer planets, but even the terrestrial ionosphere is incompletely understood. The intent of this paper is to give a review of the fundamental sources of ionospheric layers and delineate the unique changes in energy inputs into the upper atmosphere-ionosphere and their consequences for the different atmosphere-laden solar system bodies. This will encompass a survey of seven planets (Venus, Earth, Mars, Jupiter, Saturn, Uranus, Neptune) and 5 moons with substantial ionospheres (the moons of Jupiter Io, Europa, Ganymede, and Callisto, as well as Saturn's moon Titan.).

\section{Basic energy inputs and ionization layer production}

The ionizing sources that prevail throughout the solar system are: solar EUV radiation, solar wind ionized particles, interplanetary meteoroids, and energetic charged particles produced by solar wind and/or magnetosphere induced electric fields. The solar radiation and interplanetary dust particles deposit their energy and react directly with the upper atmospheres of all atmosphere-surrounded bodies. However the solar wind can have a direct atmospheric interaction only for magnetic field-free planets like Venus and some regions of Mars. For magnetized planets the solar wind and its imbedded magnetic field has an indirect impact through the production of strong magnetospheric electric fields induced by the penetration of the solar wind dynamo electric field (i.e., the solar wind being a conducting medium moving across the interplanetary magnetic field generates a dynamo electric field across solar system magnetospheres) and by connection of the planetary magnetic field with the interplanetary magnetic field. For the magnetic planets, these processes produce energetic charged particles that in turn impact the planetary atmospheres. The fast rotation of the outer planets, in particular Jupiter and Saturn, itself also produces energized plasma from the ionization of the planetary ionosphere production and/or gases emitted from their moons.

The diverse energy inputs lead not only to the production of ionospheres, but spatially varying atmospheric and ionospheric heating that drive ionospheric motions and upper atmospheric winds causing redistributions of the ionospheric morphology. The sources of ionization vary in magnitude and spatial extent from atmosphere to atmosphere and the resulting ionospheres are unique to each planetary and lunar atmosphere in the solar system. The results are further dependent on the atmospheric composition which varies from the dominance of $\mathrm{N}_{2}$ and $\mathrm{O}_{2}$ at Earth, through the $\mathrm{CO}_{2}$ atmospheres of Venus and Mars, the hydrogen atmospheres of the giant planets and the varied atmospheric complexities of planetary moons arising from gaseous emissions of water or volcanic gases.

In all cases the ionosphere and upper atmosphere comprise one integral system. A comprehensive list of processes that can control an ionosphere consists of: Solar radiation; Neutral atmosphere composition topology; Photochemistry; Meteoroid ionization (dependent on ablation of interplanetary micro-particles, ambient atmosphere and ionosphere and photochemistry), Magnetospheric coupling (through energetic particle production, joule heating, magnetosphere reservoir of ionospheric particles); Solar wind coupling (precipitation of SW ionization, induced magnetic fields and electric field accelerations), Atmospheric drag (winds, tides, waves).

\section{Nominal background ionospheres}

The nominal ionospheric layers, corresponding to the electron density (or total ion density) resulting from the photoionization by solar EUV are well established throughout the 
solar system from theory. This understanding evolved from comprehensive global measurements of ionospheric ion composition of the layers at Earth, Pioneer Venus Orbiter measurements in the topside of the Venus ionosphere, from the descent of two Viking lander probes at Mars and from radio occultation measurements of vertical electron density profiles made on all planetary spacecraft missions.

The general origin of ionospheres is best described by considering a typical vertical profile of the terrestrial ionosphere (Figure 1, adapted from Rich, 1985) and the effects of photochemistry. With decreasing altitude, ionization is produced by the more energetic wavelengths of the solar radiation with the ionization peaks occurring near the altitude with maximum atmospheric absorption of the shorter wavelength bands. It leads to ionization density peaks corresponding to the D and E layers. Molecular ions, produced by photoionization of atmospheric species, dominate the ion composition in these layers. The ions are in photochemical equilibrium with the atmosphere, as is also the case for the higher layer, F1, where atomic ions begin to appear as the neutral molecular species drop off with increasing altitude. In addition, from the E region peak to below $80 \mathrm{~km}$ metal ion species are also present (as seen in every ion composition measurement from sounding rockets, e.g., Grebowsky and Aikin 2002) as are layers of neutral metal species (seen from ground based LIDAR measurements). These are the products of ablated metal atoms from incoming meteoroids. The metal ions are produced by photoionization and charge exchange of the ablated neutral atoms with ambient molecular ions. The metal ions have low ionization potentials and long lifetimes.

The main terrestrial ionospheric layer, the F2 peak, is no longer in photochemical equilibrium. The atmosphere is no longer dense enough to prohibit vertical diffusion of the ionization. Diffusion plays a role in the formation of the layer structure. At night, where solar EUV photoionization is absent and only backscattered radiation is available for photoproduction, $\mathrm{F}$ region ionization is supplied from transport of ionization from the dayside or downward transport of ionization that was transported up during the day (see Figure 1). Metal ion species are still measured at night at the lower altitudes because of their long chemical lifetime.

This simple picture of the ionosphere, however, is altered with motions induced in the neutral atmosphere and ionosphere by the energy inputs from the solar wind and magnetosphere, particularly in the form of auroral energetic particle precipitations and heating effects and transport of the ionospheric plasma by the solar wind electric field penetrating to low altitudes. These motions, that vary in response to changes in solar activity, lead to a very complex ionospheric morphology (see Figure 2). We are beginning to model successfully many of the complexities for Earth, but for other solar system bodies we do not yet have the measurements to even begin to understand the complexities. The Earth's

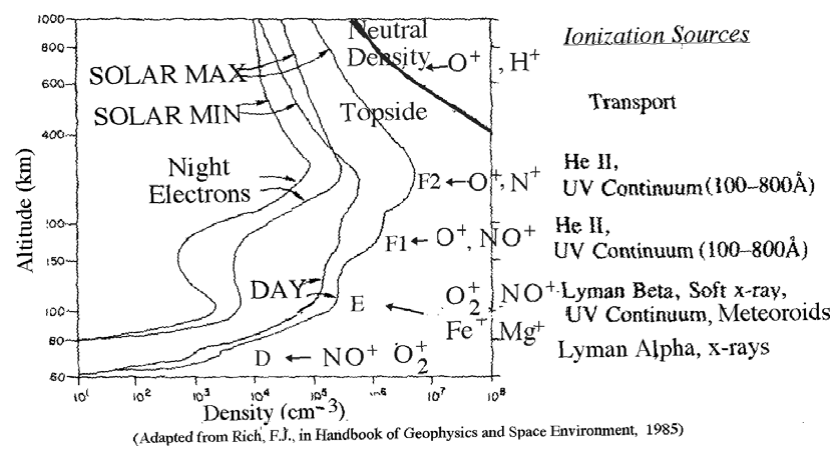

Figure 1. Description of ionospheric layers on Earth, showing the composition of major ions. 
internal magnetic field, observed auroral heating effects, and atmospheric/ionospheric variations puts it in a classification with the outer giant planets that have intrinsic magnetic fields. On the other hand, magnetic field-free planets Mars and Venus have novel ionospheric processes operating. We have only a few spacecraft that sampled the outer planets and these are mostly from remote observations, with the exception of Cassini, which is currently sampling directly the ionosphere of Titan at Saturn. The Mars ionosphere has and is being explored remotely by Mars Explorer (MEX) and Mars Global Surveyor (MGS). However, there are extensive in situ ionospheric measurements of Venus from Pioneer Venus Orbiter (PVO), and also a few in situ ionospheric measurements at Mars from the Viking landers that provide glimpses of the types of processes operating in magnetic field-free atmospheres.

\section{Magnetic field-free atmospheres: Mars and Venus}

The first upper atmosphere/ionosphere difference in moving from Earth to the other terrestrial planets of Mars and Venus is the change of atmospheric composition from nitrogen/oxygen to carbon dioxide dominances. Another distinguishing factor from Earth is the absence of a global magnetic field, although Mars has localized pockets of remnant magnetic fields (Connerney et al. 2001) near its surface. Also Mars and Venus have different solar radiation influxes than Earth. The incident solar EUV flux is $\sim 4$ times lower for Mars, resulting in a much weaker ionosphere at Mars compared to Venus. (Mendillo et al. 2003, showed that on average the dayside peak electron density of all planetary ionospheres decreases with increasing distance from the Sun as expected for the EUV flux drop off). One consequences of a weakened ionosphere for Mars and the increased scale height of the atmosphere due to Mars's low gravity is that its topside ionospheric pressure is not sufficient to protect the topside ionospheric plasma from being stripped off by the solar wind. On the other hand, Pioneer Venus observations have shown that during solar maximum the ionospheric pressure is high enough to form a barrier to the solar wind that is far above the collision-dominated, photochemical ionosphere region. Figure 3 shows dayside observations on both Venus and Mars (the

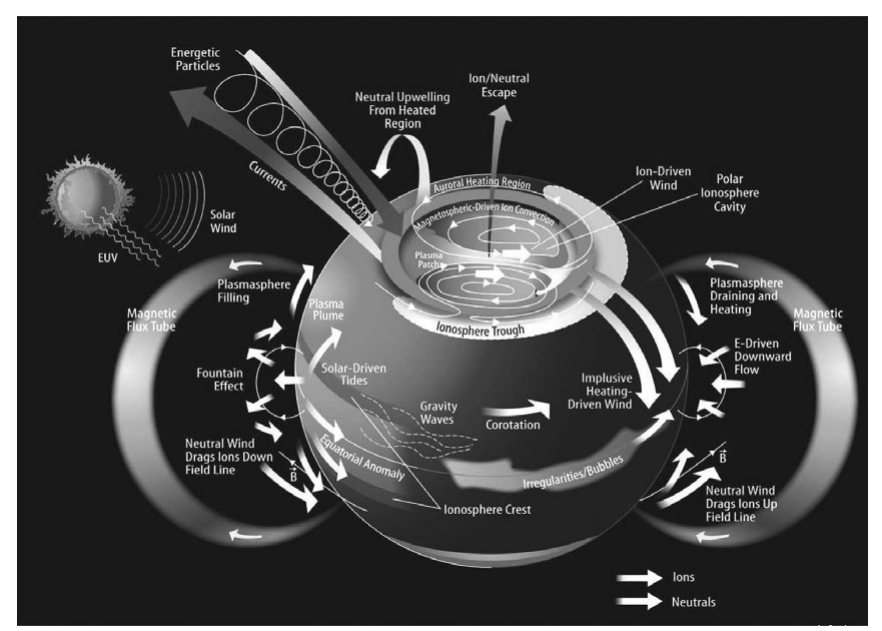

Figure 2. Complexity of upper atmosphere/ionosphere structures at Earth. 

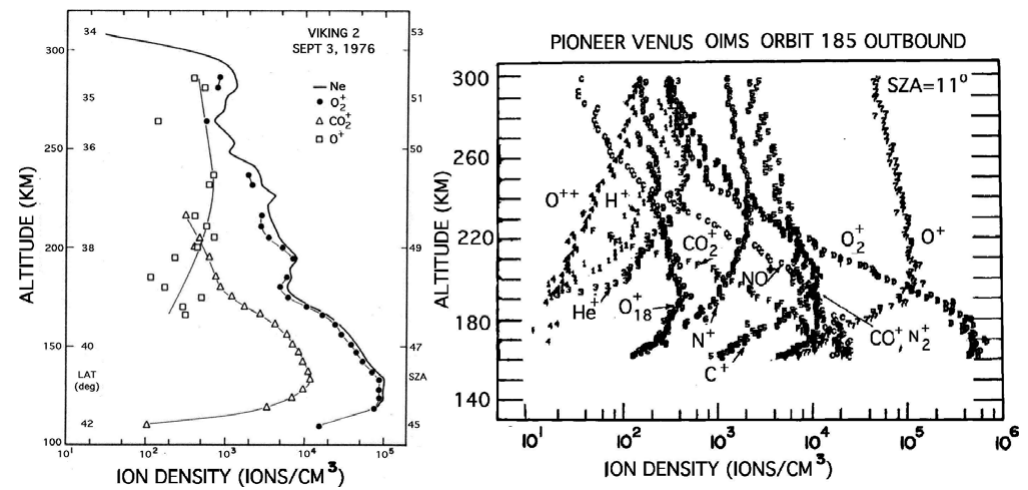

Figure 3. Measurements of ion composition (Hanson et al. 1977) from the entrance of one of the two Viking landers at Mars and one Pioneer Venus Orbit from the Orbital Ion Mass Spectrometer (Taylor et al. 1980).

former obtained from the Planetary Data System Pioneer Venus observations, the later from Hanson et al. 1977). The main $\mathrm{O}_{2}^{+}$layers on both planets are chemical equilibrium layers as on Earth, but there is a transition into an $\mathrm{O}^{+}$dominant ionosphere on Venus. The solar wind influence on Mars readily strips the ions produced by photoionization on the topside ionosphere. At Venus however, the boundary of penetration of the shocked solar wind influence (the ionopause) reaches as high as $1000 \mathrm{~km}$ at solar maximum. During solar minimum, when solar EUV and photoproduction is reduced, the Venus ionosphere becomes analogous to the Mars situation in that the solar wind electrodynamics results in stripping off of ionization deep in the ionosphere - the ionopause moves in to 200 $300 \mathrm{~km}$ (Kliore and Luhmann 1991).

Earth has a substantial nightside ionosphere because of transport of photoionizedproduced ions, that corotate with the Earth, from the dayside. However, Venus has a very slow rotation rate, so it was somewhat surprising when a substantial nightside ionosphere was observed. The clue to its origin was confirmed by the pioneer Venus Orbiter Retarding Potential Analyzer observations of horizontal ion drifts, which reached supersonic speeds, from the day into the night (Knudsen et al. 1980) - the nightside ionosphere was being supplied from the day, the motions driven by the pressure gradient between the nightside chemical sink and the dayside photoproduction source. There were periods, however, during the PVO mission when the solar wind dynamic pressure was high and the nightside ionosphere became vanishingly weak. Indeed observations near solar minimum, just before the end of the mission, found the nightside ionosphere to be in a persistently low density state similar to the solar maximum, high solar wind dynamic pressure events. The topside ionosphere often vanished. On Mars on the other hand radio occultation measurements have indicated in general a very weak, variable, or vanishing nightside ionosphere. These measurements are consistent with the interpretation that there is competition between photoproduction of the dayside ionosphere and stripping away of the ionization by the solar wind. The magnitude of the solar wind dynamic pressure relative to the ionospheric pressure controls the nightside ionosphere. Mars is always in the state where the solar wind dominates. Venus is in that state only during solar minimum or under enhanced solar wind conditions.

Current understanding of the Venus nightside is shown in Figure 4 (adapted from Brace and Kliore, 1991). The nightside ionosphere is formed by the transport of $\mathrm{O}^{+}$from the day that then falls under the force of gravity to chemically produce an $\mathrm{O}_{2}^{+}$layer. There are also distinctive holes in the ionosphere that are related to the draping of the 


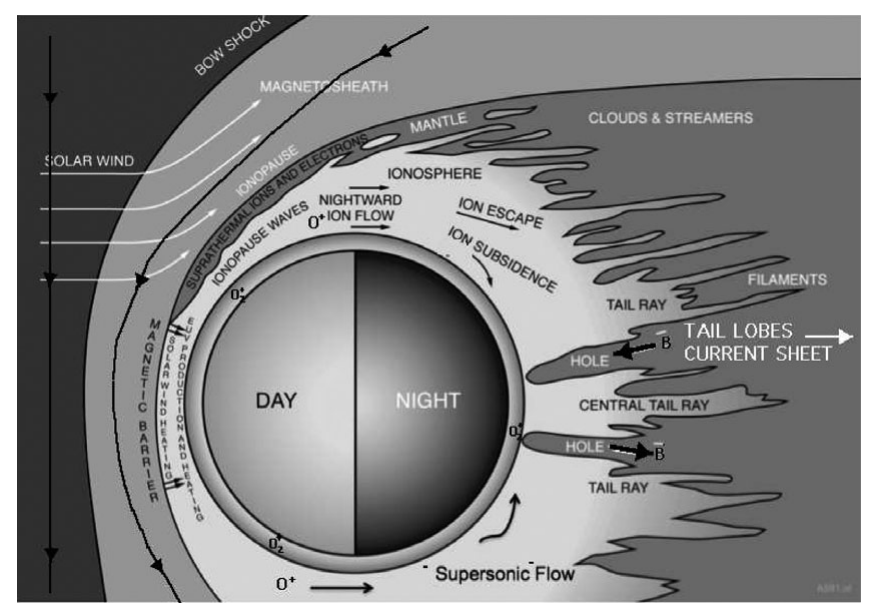

Figure 4. Model of the Venus plasma environment (Adapted from Brace and Kliore 1991).

interplanetary magnetic field in the ionosphere. Although there were a large number of PVO passages through these regions, their origin is still not fully understood. During solar minimum or periods of high solar wind dynamic pressure all the topside ionosphere during the day is ripped off by the solar wind electric field and transported energetically down the Venus tail eliminating the nightside ionosphere transport source. However, even under these conditions an $\mathrm{O}_{2}^{+}$layer is still often present at night - this is attributed to the precipitation of solar wind electrons, energetic enough to ionize the atmosphere.

The depleted nightside ionosphere of Venus appears to be a close analogy to the Martian nightside state. Overall the solar wind-forcing accelerates most of the topside ionosphere production on the dayside down the tail, rather than allow it to subside at night due to gravity, which is less than at Venus. The exceptions may occur in the magnetic anomaly regions, since these magnetic fields when closed, act as retainers for ionospherically produced plasma that will rotate with these field lines. Observations from Mars Express have seen UV auroral emissions (Bertaux et al. 2005) in the atmosphere and kev energetic ions and precipitating particles in the cusp regions of the magnetic fields where the planetary field connects with the interplanetary magnetic field. This is a source for ionospheric electrons and auroral emissions (Brain et al. 2006) as occurs in the Earth's polar cap.

Although we have a general concept of the global variations of the ionospheres at Mars and Venus, we are missing vital measurements. The observations thus far have not enabled us to develop global empirical models of the ionosphere, and measurements of the atmospheric dynamical control of the ionosphere are totally lacking as is the global consequences of the magnetic anomalies on Mars.

\section{The giant planets}

\subsection{Jupiter}

Jupiter is the largest planet in the solar system with a radius 11.2 times that of Earth. The planet possesses a strong magnetic field that results in a significant magnetosphere. There is interaction between many of Jupiters 63 moons and its magnetosphere. Jupiter's atmosphere is $89.8 \% \mathrm{H}_{2}$ and $10.2 \% \mathrm{He}$, with trace amounts of $\mathrm{CH}_{4}$ and $\mathrm{NH}_{3}$. Dissociation of methane leads to the production of hydrocarbons including ethane and acetylene. 


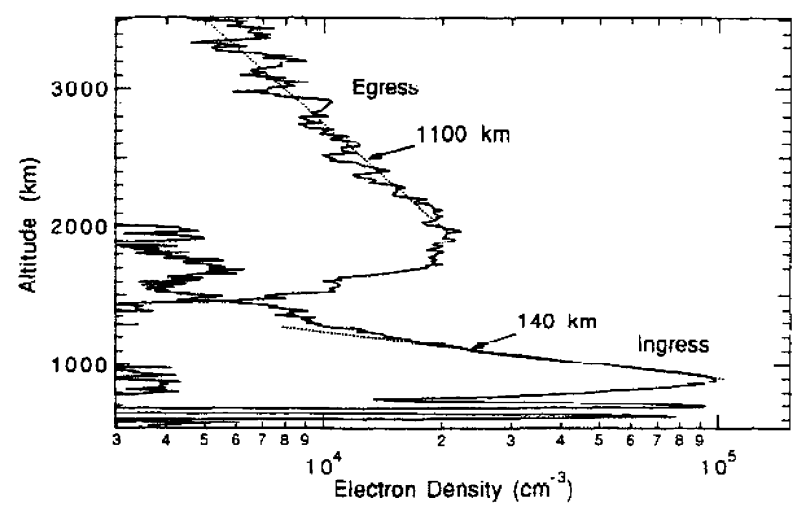

Figure 5. Jupiter ionosphere ingress and egress electron density profiles (Hinson et al. 1997).

Hydrocarbons are restricted to the lower and middle atmosphere. Helium falls off with altitude so that $\mathrm{H}_{2}$ and $\mathrm{H}$ are the chief component of the thermosphere and exosphere.

The ionosphere has been measured using radio occultation experiments on both the Pioneer and Voyager spacecraft. Figure 5 shows electron density profiles from Voyager for both an ingress and egress measurement (Hinson et al. 1997). The ingress measurement was taken when the solar zenith angle was 89 degrees in the evening as opposed to the egress measurement taken for a solar zenith angle of 91 degrees in the morning. Peak electron densities are $10^{5} \mathrm{~cm}^{-3}$ and $2 \times 10^{4} \mathrm{~cm}^{-3}$ for the two cases. Altitudes for the peaks are 900 and $2000 \mathrm{~km}$. Ingress measurements are consistent with daytime photoionization equilibrium conditions both from the altitude and magnitude standpoint. The egress profile is more difficult to explain in both altitude and magnitude. It is important to keep in mind that conditions are sunrise and that the Jupiter ionosphere is strongly influenced by energetic electrons and other charged particles from Jupiters magnetosphere. Between 600 and $800 \mathrm{~km}$ altitude on the ingress measurement there are several narrow ionization regions. One possible explanation is that the origin of these layers is the focusing of long-lived metallic ions, whose origin is meteoric debris, by wind shears moving across Jupiters magnetic field. The possibility that metal ions are an important constituent of the Jupiter ionosphere has been considered by Kim et al. (2001).

\subsection{Saturn}

With a similar atmosphere and size as Jupiter one would expect the ionosphere of Saturn to have an electron density and ion composition as Jupiter. In Figure 6 are presented a series of electron density profiles taken with the radio occultation experiment on the Cassini spacecraft (Moore et al. 2006). The peak electron densities are in the $10^{3}$ to

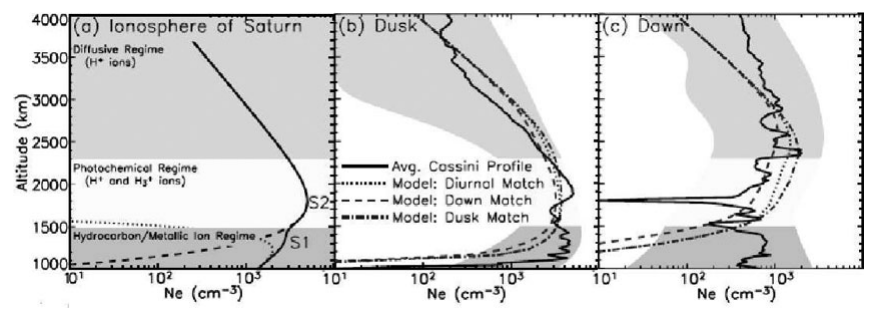

Figure 6. Saturn average electron density profiles and profiles for dusk and dawn. Results from a model are also shown for comparison. (Adapted from Moore et al. 2006.) 


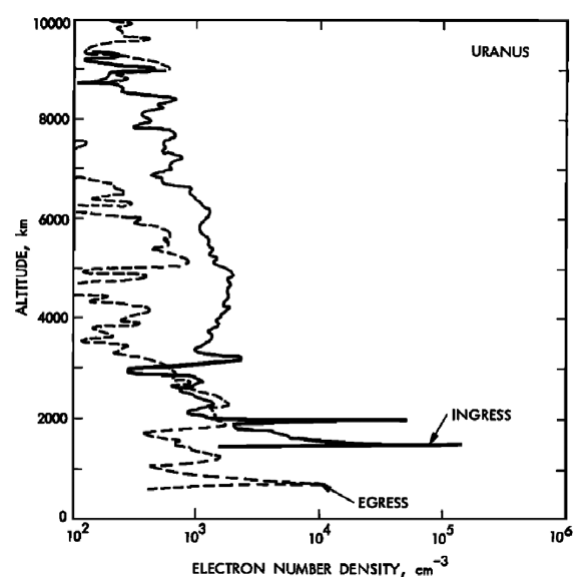

Figure 7. Electron density ingress and egress profiles for Uranus (Lindal et al. 1987).

$10^{4} \mathrm{~cm}^{-3}$. In contrast the Jupiter maximum electron densities are in the $10^{4}$ to $10^{5} \mathrm{~cm}^{-3}$. This order of magnitude difference in number density cannot be explained simply as the difference in solar radiation between Jupiter and Saturn. The explanation for the difference is that the Saturn ionosphere has a predominance of molecular ions compared with Jupiter where $\mathrm{H}^{+}$is the dominant ion. Atomic ions recombine with electrons by radiative recombination as opposed to molecular ions that recombine by dissociative recombination. Radiative recombination is slower than dissociative recombination by several orders of magnitude. The added neutral constituent that leads to an increase in recombination rate is water, which is moved from Saturn's rings. Reactions such as

$$
\mathrm{H}^{+}+\mathrm{H}_{2} \mathrm{O} \rightarrow \mathrm{H}_{2} \mathrm{O}^{+}+\mathrm{H}
$$

and

$$
\mathrm{H}_{2} \mathrm{O}^{+}+\mathrm{H}_{2} \mathrm{O} \rightarrow \mathrm{H}_{3} \mathrm{O}^{+}+\mathrm{OH}
$$

lead to the molecular ion $\mathrm{H}_{3} \mathrm{O}^{+}$. Examination of the profiles in Figure 6 shows regions of electron density depletion. These regions can be explained by sudden water influxes from the rings. Saturn possess a magnetosphere and exhibits aurora at both poles.

\subsection{Uranus}

Unlike Jupiter and Saturn, Uranus is covered by a deep ocean of methane, water and ammonia. These compounds are important components of the lower atmosphere. Photodissociation of methane and subsequent chemical reactions leads to a variety of hydrocarbon compounds including ethane, and acetylene. Both hydrogen and helium are the principal constituents of the thermosphere with helium falling off by diffusive separation so that the bulk of the thermosphere is $\mathrm{H}$ and $\mathrm{H}_{2}$. The planet has an atmosphere that extends more than 2 planetary radii. The ionosphere of Uranus was measured using the radio occultation experiment on Voyager 2. A sample pair of electron density profiles is shown in Figure 7 (Lindal et al. 1987). For the most part the electron densities are in the $10^{3}$ to $10^{4} \mathrm{~cm}^{-3}$ range. This is consistent with results from Saturn, but the densities are less than Jupiter. This result is consistent with the requirement for an ion composition with molecular ions as the principal constituent so that the chief electron loss process is dissociative recombination leading to lower electron densities. This implies that water is a component of the atmosphere. 


\subsection{Neptune}

The atmosphere of Neptune is similar to that of Uranus with $\mathrm{He}, \mathrm{H}_{2}$, and $\mathrm{H}$ principal constituents of the upper atmosphere. Like Uranus, Neptune possess a magnetic field, giving rise to a magnetosphere. The ionosphere has been measured using the radio occultation experiment on Voyager 2 (Tyler et al. 1989). The electron density profile between 1000 and $5000 \mathrm{~km}$ decreases from $2 \times 10^{3} \mathrm{~cm}^{-3}$ at $1000 \mathrm{~km}$ to less than $100 \mathrm{~cm}^{-3}$ at $5000 \mathrm{~km}$.

\section{Jupiter's moons with ionospheres}

\subsection{Io}

Io is the closest moon to Jupiter. With a radius of 0.286 that of Earth, close to the radius of the Earth's moon, and a surface gravity only $0.183 \mathrm{~g}$ of Earths value, the moon would not be expected to possess an atmosphere. However, because of the moons proximity to Jupiter and the composition of the moon, a rocky core with a silicate mantle and surface, strong tidal forces give rise to volcanism. The large number of active volcanoes produce a large variety of sulfur compounds. Some eruptions reach $500 \mathrm{~km}$ above the surface. As a result Io has an atmosphere of mostly $\mathrm{SO}_{2}$ with a surface pressure of $10^{-12}$ bar. There is an ionosphere with a peak electron density of $3 \times 10^{5} \mathrm{~cm}^{-3}$. Solar radiation is only 0.04 that of the amount striking the Earth. Precipitation of energetic charged particles from Jupiters magnetosphere is an important source of ionization. The effect of the Jupiter magnetosphere is evident in the existence of an aurora zone on Io. The zone is centered near Io's equator. The Jupiter magnetic field lines passing through the moon are a source of ionizing particles for the atmosphere, and aid in the escape of sulfur compounds into the Jovian magnetosphere.

\subsection{Europa}

Jupiter's moon Europa has a radius that is 0.245 that of Earth. Compared to Earth its surface gravity is $0.134 \mathrm{~g}$. It has been found that Europa possesses an atmosphere composed of molecular oxygen (Hall et al. 1995). The origin of the oxygen atmosphere is the fact that the surface of Europa is covered with ice and also subject to the bombardment by charged energetic particles and electrons from Jupiters magnetosphere. The impact of these particles causes sputtering of water from the surface. Further interaction of the particles with the freed water dissociates the water to produce free oxygen, which is less reactive than the hydrogen produced by the water dissociation reaction. The surface pressure is $1 \times 10^{-11}$ bar.

The measurements of the Europa electron density are based on radio occultation data from the Galileo spacecraft (Kliore et al. 1997). The entry point for the occultation occurs on the dayside of the moon. This is also on the ram side for the entry of magnetospheric particles. On this side there is a significant ionosphere with a surface electron density of $2 \times 10^{4} \mathrm{~cm}^{-3}$. The plasma scale height is $240 \pm 40 \mathrm{~km}$. The exit occultation for this measurement occurs on the nightside of the moon. For this situation there is no measurable ionosphere. The comparison of these electron density profiles indicates that the ionosphere is the result of photoionization of the atmospheric $\mathrm{O}_{2}$ and the entry of magnetospheric particles. Based on the neutral atmospheric measurements of $\mathrm{O}_{2}$ as the major constituent the principal ion is $\mathrm{O}_{2}^{+}$.

\subsection{Ganymede}

Ganymede is the largest of Jupiters moons with a radius that is 0.413 of Earth. The acceleration of gravity is $0.146 \mathrm{~g}$ that of Earth. Ganymede is very similar to Europa 
in its covering of water ice and being subject to bombardment by energetic charged particles and electrons from the Jovian magnetosphere. This bombardment gives rise to a molecular oxygen atmosphere with a surface pressure of $1 \times 10^{-14}$ bar.

There is an ionosphere with the peak density between 400 and $2500 \mathrm{~cm}^{-3}$. Ganyede is unique because it possesses its own magnetic field and a weak magnetosphere. Ions and electrons from the surface are swept along the field lines to form the magnetosphere together with input from Jupiter's magnetosphere. In addition to its intrinsic magnetic field, Ganymede possesses an induced magnetic field.

\subsection{Callisto}

The Jovian moon Callisto is the fourth in distance from the planet. Its size is approximately the same as Mercury, with a radius of 0.378 that of Earth and an acceleration due to gravity of $0.126 \mathrm{~g}$. The moon is not subject to orbital induced tidal forces and is covered with carbon dioxide ice. Sublimation of $\mathrm{CO}_{2}$ from the surface has given rise to a $\mathrm{CO}_{2}$ atmosphere with a surface pressure of $7.5 \times 10^{-12}$ bar and a surface number density of $4 \times 10^{8} \mathrm{~cm}^{-3}$.

As a result of photoionization of the $\mathrm{CO}_{2}$ atmosphere Callisto possesses an ionosphere. The maximum electron density is $1.74 \times 10^{4} \mathrm{~cm}^{-3}$ at an altitude of $47.6 \mathrm{~km}$. The plasma scale height is $49 \mathrm{~km}$. The ionosphere corotates with the planet, which always presents the same face to Jupiter.

\section{Titan}

Titan is the largest moon of the Saturn system and the only one to possess an atmosphere. The radius of the moon is 0.4 that of the Earth with a surface gravity of $0.14 \mathrm{~g}$. Although the surface pressure is $1.4 \mathrm{bar}$, the surface temperature is only $94 \mathrm{~K}$. The composition of the atmosphere is $98.4 \% \mathrm{~N}_{2}$ and $1.6 \%$ methane with trace amounts of more complex hydrocarbons. The duration of the diurnal cycle is 30 years.

The daytime ionosphere has a peak electron density of $10^{4} \mathrm{~cm}^{-3}$ in spite of the fact that the solar ionizing radiation is only $1 \%$ of the flux reaching Earth. In addition there is the ionization by electrons and ions associated with Saturns magnetosphere. The importance of this ionization source is demonstrated by the substantial nighttime ionosphere with a peak ionization of $10^{3} \mathrm{~cm}^{-3}$ over an altitude range of 1000 to $1400 \mathrm{~km}$. Based on comparison with the daytime ionosphere the magnetosphere ionization source is about $1 \%$ of the daytime photoionizatioin source. Cosmic radiation is a small additional ionization source operative at altitudes below the daytime and nighttime ionization peaks. The principal positive ion of the ionosphere is $\mathrm{HCNH}^{+}$. Secondary ions include $\mathrm{C}_{2} \mathrm{H}_{5}^{+}, \mathrm{C}_{3} \mathrm{H}_{5}^{+}$, and $\mathrm{CH}_{5}^{+}$. Negative ions have been measured at $960 \mathrm{~km}$. The negative ions can be arranged by mass range: 10 to $30 \mathrm{amu}$, candidate ion species $\mathrm{CN}^{-} ; 30$ to $50 \mathrm{amu}$ candidate ion species $\mathrm{NCN}^{-}, \mathrm{HNCN}^{-}, \mathrm{C}_{3} \mathrm{H}^{-} ; 50$ to $80 \mathrm{amu}$ candidate ion species $\mathrm{C}_{5} \mathrm{H}_{5}^{-}, \mathrm{C}_{6} \mathrm{H}^{-}$, $\mathrm{C}_{6} \mathrm{H}_{5}^{-} ; 80$ to 110 amu candidate ion species polynes, nitriles, PAH; 110 to $200 \mathrm{amu}$ candidate ion species polynes, nitriles, PAH; > 200 amu candidate ion species polynes, nitriles, PAH. Positive ion composition measurements (Waite et al. 2007) have shown that the ion $\mathrm{C}_{6} \mathrm{H}_{7}^{+}$is present. This ion can be correlated with the presence of large concentrations of benzene, $\mathrm{C}_{6} \mathrm{H}_{6}$, in the thermosphere. Dissociative electron-ion recombination of $\mathrm{C}_{6} \mathrm{H}_{7}^{+}$ by the reaction

$$
\mathrm{C}_{6} \mathrm{H}_{7}^{+}+\mathrm{e}^{-} \rightarrow \mathrm{C}_{6} \mathrm{H}_{6}+\mathrm{H}
$$

yields benzene. 


\section{Conclusions}

All bodies in the solar system are subject to solar radiation, which includes ultraviolet and $\mathrm{x}$ radiation capable of ionizing atmospheric gases of those bodies possessing atmospheres, forming ionospheres. Those planets and moons not possessing an intrinsic magnetic field, including Venus and Mars, have their atmospheres subject to the ionizing radiation of the charged energetic particles that comprise the solar wind. The interaction of the solar wind with the intrinsic magnetic field of planets such as Earth and Jupiter produces a magnetosphere for each of these bodies. Electrons in magnetospheres are accelerated to higher energies. Precipitation of these electrons into a planet's atmosphere gives rise to an auroral zone. Moons of magnetospheric planets are subject to energetic particles, which can modify the moons' ionospheres. Moons in this category are the Jovian moons of Io, Europa, Ganymede, and Callisto as well as Saturn's moon Titan. Although we now understand which bodies have ionospheres and how they are formed, the detailed behavior of these ionospheres is unknown. Future ionospheric missions will not only fill in details, but may uncover new phenomena.

\section{References}

Bertaux, J.-L., Leblanc, F., Witasse, O., Quemerais, E., Lilensten, J., Stern, S. A., Sandel, B., \& Korablev, O. 2005, Nature, 435, 790

Brace, L. H. \& Kliore, A. J. 1991, Space Science Reviews, 55, 81

Brain, D. A., et al. 2006, Geophys. Res. Lett., 33, 1201

Connerney, J. E. P., Acuña, M. H., Wasilewski, P. J., Kletetschka, G., Ness, N. F., Rème, H., Lin, R. P., \& Mitchell, D. L. 2001, Geophys. Res. Lett., 28, 4015

Grebowsky, J. M. \& Aikin, A. C. 2002, Meteors in the Earth's atmosphere. Edited by Edmond Murad and Iwan P. Williams. Publisher: Cambridge, UK: Cambridge University Press, 2002., p.189, 189

Hall, D. T., Strobel, D. F., Feldman, P. D., McGrath, M. A., \& Weaver, H. A. 1995, Nature, 373,677

Hanson, W. B., Sanatani, S., \& Zuccaro, D. R. 1977, J. Geophys. Res., 82, 4351

Hinson, D. P., Flasar, F. M., Kliore, A. J., Schinder, P. J., Twicken, J. D., \& Herrera, R. G. 1997, Geophys. Res. Lett., 24, 2107

Kliore, A. J. \& Luhmann, J. G. 1991,J. Geophys. Res., 96, 21281

Kliore, A. J., Hinson, D. P., Flasar, F. M., Nagy, A. F., \& Cravens, T. E. 1997, Science, 277, 355

Kim, Y. H., Pesnell, W. D., Grebowsky, J. M., \& Fox, J. L. 2001, Icarus, 150, 261

Knudsen, W. C., Spenner, K., Miller, K. L., \& Novak, V. 1980, J. Geophys. Res., 85, 7803

Lindal, G. F., Lyons, J. R., Sweetnam, D. N., Eshleman, V. R., \& Hinson, D. P. 1987, J. Geophys. Res., 92, 14987

Mendillo, M., Smith, S., Wroten, J., Rishbeth, H., \& Hinson, D. 2003, J. Geophys. Res., 108, 1432

Moore, L., Nagy, A. F., Kliore, A. J., Müller-Wodarg, I., Richardson, J. D., \& Mendillo, M. 2006, Geophys. Res. Lett., 33, 22202

Rich, F. J. 1985, Ionospheric Physics: Chapter 9 in the Handbook of Geophysics and the Space Environment. Edited by A. S. Jursa. Publisher: Air Force Geophysics Laboratory: United States Air Force, 1985

Taylor, H. A., Brinton, H. C., Bauer, S. J., Hartle, R. E., Cloutier, P. A., \& Daniell, R. E. 1980, J. Geophys. Res., 85, 7765

Tyler, G. L., et al. 1989, Science, 246, 1466

Waite, J. H., Young, D. T., Cravens, T. E., Coates, A. J., Crary, F. J., Magee, B., \& Westlake, J. 2007, Science, 316, 870 


\section{Discussion}

BochSLER: Mars Express has recently observed an increase by 1 order of magnitude in the ion outflow from the Martian ionosphere (Futaana et al., PSS, 2008) during an SEP event in December 2006. Would this affect the communications in a similar way to the Earth?

Grebowsky: Ion outflow would have no effect on communications unless SEP was associated with the production of an ionospheric density enhancement.

GIRISH: 1. The galactic cosmic ray effects in night-side ionospheres of Mars and Venus? 2. The effect of very slow rotation (243 days) of Venus on its atmospheric dynamics?

Grebowsky: 1. Measurements of cosmic ray effects on ionospheres of Mars and Venus have not been made, but models have included ionization by cosmic rays. 2. Venus' atmosphere actually rotates faster than planet-i.e. it super rotates.

JARDINE: Can you say a little about observations of Enceladus?

Grebowsky: Enceladus sporadically has intense emissions of water and ice. These ongassed products are a significant source of Saturn's magnetospheric plasma. 